\title{
A detection of benzimidazole resistance- associated SNPs in the isotype-1 $\beta$-tubulin gene in Haemonchus contortus from wild blue sheep (Pseudois nayaur) sympatric with sheep in Helan Mountains, China
}

\author{
Dong-dong Shen ${ }^{1}$, Zhi-wei Peng ${ }^{1}$, Min Hư ${ }^{3}$, Zong-ze Zhang ${ }^{3}$, Zhi-jun Hou ${ }^{1,2^{*}}$ (D) and Zhen-sheng Liu ${ }^{1,2}$
}

\begin{abstract}
Background: Benzimidazole (BZ) resistance is an increasingly serious problem due to the excessive use of this anthelmintic for controlling Haemonchus contortus, which is one of the major gastrointestinal nematodes infecting small ruminants worldwide. Three known single nucleotide polymorphisms (SNPs), F167Y (TAC), E198A (GCA) and F200Y (TAC), in the isotype-1 $\beta$-tubulin gene of $\mathrm{H}$. contortus are associated with BZ resistance. Comprehending the spread and origins of BZ resistance-associated SNPs has important implications for the control of this nematode.

Results: Twenty-seven adult $H$. contortus were harvested from wild blue sheep (Pseudois nayaur), small wild ruminants sympatric with domestic ruminants, inhabiting the Helan Mountains, China, to monitor the status of BZ resistance. In addition, 20 adult $H$. contortus from domestic sheep sympatric with this wild ruminant and 36 isotype$1 \beta$-tubulin haplotype sequences of H. contortus (two of these haplotypes, E198A3 and E198A4, possessed resistance-associated SNP E198A (GCA) from domestic ruminants in eight other geographical regions of China were used to further define the origins of BZ resistance-associated SNPs within the worms collected from blue sheep. The BZ resistance-associated SNP E198A was detected, whereas SNPs F167Y (TAC) and F200Y (TAC) were not found within the worms collected from blue sheep, and the frequency of homozygous resistant E198A (GCA) was 7.40\%. The evolutionary tree and network showed consistent topologies for which there was no obvious boundary among the worms from the wild and domestic hosts, and two haplotypes (E198A1 and E198A2) possessing E198A from the wild blue sheep had two different independent origins. E198A1 had the same origin with E198A3 but E198A2 had a different origin with them. Population genetic analyses revealed a low level of Fst values (ranging from 0 to 0.19749) between all H. contortus worm groups in China.
\end{abstract}

Conclusions: Results of the current study of the three BZ resistance-associated SNPs of $\mathrm{H}$. contortus from wild blue sheep suggested that only E198A (GCA) was present within the worms collected from the wild ruminants and had multiple independent origins.

Keywords: Blue sheep, Haemonchus contortus, Isotype-1 $\beta$-tubulin gene, Benzimidazole resistance, Helan Mountains

\footnotetext{
*Correspondence: houzhijundz@163.com

${ }^{1}$ College of Wildlife Resources, Northeast Forestry University, Harbin, China

${ }^{2}$ Key Laboratory of Wildlife Conservation, China State Forestry Administration,

Harbin, China

Full list of author information is available at the end of the article
}

(c) The Author(s). 2019 Open Access This article is distributed under the terms of the Creative Commons Attribution 4.0 International License (http://creativecommons.org/licenses/by/4.0/), which permits unrestricted use, distribution, and reproduction in any medium, provided you give appropriate credit to the original author(s) and the source, provide a link to the Creative Commons license, and indicate if changes were made. The Creative Commons Public Domain Dedication waiver (http://creativecommons.org/publicdomain/zero/1.0/) applies to the data made available in this article, unless otherwise stated. 


\section{Background}

Haemonchus contortus is one of the main gastrointestinal nematodes that infect small ruminants worldwide [1]. A prolific breeder, one adult female worm can produce nearly ten thousand eggs every day [2]. During this worm's parasitic period, eggs are continually expelled from the infected hosts and develop in the external environment, such as pastures to the third-stage larvae (L3 s) that can infect suitable ruminant hosts [3]. Since adults of this gastrointestinal nematode feed on the blood of hosts, the seriously infected ruminants may have some clinical presentation such as weight loss, anaemia and even death [4]. Thus, this nematode economically impacts livestock production [5].

In order to minimize the economic losses to animal husbandry caused by this nematode, benzimidazoles (BZs) have been widely used against this worm. However, the BZ resistance of $H$. contortus has become an increasingly serious problem due to excessive use of this anthelmintic [6]. Studies to date have reported that three different single nucleotide polymorphisms (SNPs) in the isotype-1 $\beta$-tubulin gene at codons 167 (TTC to TAC) [7], 198 (GAA to GㅡA) [8] and 200 (TTC to TAC) [9] are correlated with $\mathrm{BZ}$ resistance in $H$. contortus. The F200Y (TAC) which results in the replacement of phenylalanine with tyrosine seems to be the commonest SNP correlated with BZ resistance and has a high frequency in many countries [10]. Though the SNP F167Y (TAC) that results in the replacement of phenylalanine with tyrosine has also been detected to be associated with BZ resistance in many studies, it has a lower prevalence than F200Y (TAC), with an obviously restrained distribution in countries including the UK, the US, France, Canada, Argentina, and Brazil [7, 11-17]. SNP E198A (GCA), resulting in the replacement of glutamate with alanine, was also implicated in BZ resistance and has been found within two filed-derived $H$. contortus population collected from South Africa [8] and Australia [18]. More recently, the BZ resistance-associated SNPE198A (GCA) has been further examined using an in vitro selection of a $H$. contortus population containing both F200Y (TAC) and E298A (GCA) [19].

Understanding the spread and origins of BZ resistance-associated SNPs has important implications for the control of this nematode [20]. Consequently, population genetic studies of $\mathrm{BZ}$ resistance in $H$. contortus have been conducted in many countries. For instance, Zhang et al. [21] studied eight geographic populations of $H$. contortus in China and showed that SNPs E198A (GCA) and F200Y (TAC) had multiple independent origins, which revealed that $\mathrm{BZ}$ resistance associated alleles had repeatedly arisen in China.

According to a previous study [22], the wild blue sheep (Pseudois nayaur), a small wild ruminant sympatric with domestic ruminants, inhabiting the Helan Mountains, China, is also infected by $H$. contortus. Considering that $\mathrm{BZ}$ resistance has a wide distribution in China [21, 23], it is necessary to monitor $\mathrm{BZ}$ resistance within $H$. contortus populations from wild blue sheep. Therefore, the goals of this study were to (i) monitor the frequency on BZ resistance-associated alleles in $H$. contortus isolated from wild blue sheep and (ii) explore the origin of BZ resistance-associated SNPs in $H$. contortus populations from wild blue sheep.

\section{Results}

BZ resistance-associated SNP E198A (GCA), but not F167Y (TAC) and F200Y (TAC), were detected within the $H$. contortus worms isolated from wild blue sheep and domestic sheep

The partial isotype-1 $\beta$-tubulin gene sequences of all the $H$. contortus worms collected from the wild blue sheep and domestic sheep were successfully amplified using a nested PCR method. The BZ resistance-associated SNP E198A (GCA) was found within the worms collected from wild and domestic hosts, but F167Y (TAC) and F200Y (TAC) were not. As nematodes are diploid organisms, single peak represents homozygous allele and secondary peak detected means the heterozygous allele. The frequencies of heterozygous resistance at position 198 between the two worm groups from the wild and domestic hosts were 7.40 and $15.00 \%$. Homozygous resistance at position 198 was only found in the worms collected from blue sheep, and the frequency of this genotype was $7.40 \%$. Additionally, the frequencies of resistant allele at position 198 of the two worm groups from the wild blue sheep and domestic sheep were 11.11 and $7.50 \%$ (Table 1).

\section{Sequence diversity and origins of SNP E198A (GCA) in the $H$. contortus worms from wild blue sheep}

Two microliters aliquots of the genomic DNA from every worm were utilized to construct the pooled DNA samples that represented the two $H$. contortus groups collected from the wild and domestic hosts. The nested PCR product from the two pooled DNA samples was successfully amplified, cloned and sequenced to assess the genetic characterization of $H$. contortus worms collected from the wild blue sheep and domestic sheep; 27 and 20 isotype-1 $\beta$-tubulin gene sequences were obtained from the two worm groups, respectively, and 16 and 10 haplotypes, respectively, were defined in the two worm groups after sequence filtering. There were two shared haplotypes between the two worm groups, which led to a total of 24 haplotypes (GenBank accession nos. MH359364-MH359387). Among these, two haplotypes (E198A1 and E198A2) possessed BZ resistance-associated SNP E198A (GCA), and both were from the wild blue 
Table 1 Number and frequency (\%) with 95\% Cla of individual worm genotypes with "susceptible" and "resistant" single nucleotide polymorphism (SNP) and the allele frequency (\%) with 95\% Cl at codon E198A (GAA/GCA) associated with benzimidazole resistance in isotype-1 $\beta$-tubulin gene of $\mathrm{H}$. contortus worms from sympatric blue sheep and sheep in Helan Mountains, China

\begin{tabular}{llllll}
\hline Hosts & \multicolumn{4}{l}{ Number of genotype $^{\mathrm{b}}$ and frequency (\%) with 95\% Cl } & Frequency ${ }^{c}(\%)$ with 95\% Cl for codon E198A \\
\cline { 2 - 6 } & Hs-198 & Hr-198 & Het-198 & Susceptible & Resistant \\
\hline Sheep & $17(85.00,63.96 \sim 94.76)$ & $0(0.00,0.00 \sim 16.11)$ & $3(15.00,5.24 \sim 36.04)$ & $(92.50,80.14 \sim 97.42)$ & $(7.50,2.58 \sim 19.86)$ \\
Blue sheep & $23(85.20,67.52 \sim 94.08)$ & $2(7.40,2.06 \sim 23.37)$ & $2(7.40,2.06 \sim 23.37)$ & $(88.89,77.81 \sim 94.81)$ & $(11.11,5.19 \sim 22.19)$ \\
\hline
\end{tabular}

${ }^{\mathrm{a}} \mathrm{Cl}$ Confidence interval

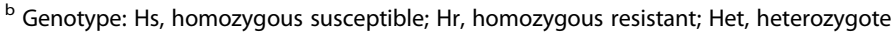

c The allele frequency for codon E198A (\%) was calculated using as below: Susceptible of sheep $=(\mathrm{Hs}-198 \times 2+\mathrm{Het}-198) /(20 \times 2)$. Resistant of sheep $=(\mathrm{Hr}-198 \times 2+$ Het-198) $/(20 \times 2)$. Susceptible of blue sheep $=(\mathrm{Hs}-198 \times 2+$ Het-198) $/(27 \times 2)$. Resistant of blue sheep $=(\mathrm{Hr}-198 \times 2+\mathrm{Het}-198) /(27 \times 2)$

sheep H. contortus worms. A high level of haplotype diversity (0.952 and 0.926) was present in the two worm groups, and the nucleotide diversity was 0.04172 and 0.03925 , respectively (Table 2).

Another 36 isotype- $1 \beta$-tubulin haplotype sequences of $H$. contortus (two of these haplotypes, E198A3 and E198A4, possessed resistance-associated SNP E198A (GCA) from domestic ruminants in eight other geographical regions of China were used to further detect origins of the two haplotypes (E198A1 and E198A2) possessing resistance-associated SNP E198A (GCA). The evolutionary tree (Fig. 1) based on Bayesian inference (10 million generations) was hypothesized using the 60 isotype- $1 \beta$-tubulin gene haplotype sequences of $H$. contortus. And the dendrogram showed that the haplotypes from the wild and domestic hosts were randomly dispersed among the branches of the phylogenetic tree. In addition, the topology of the BI tree showed (Fig. 1) that the two haplotypes possessing resistance-associated SNP E198A (GCA) from the wild blue sheep were distributed in two distinct clades (Clade 1 and Clade 2). Moreover, Clade 2 was a distinct clade with high posterior probability $(\mathrm{BPP}=100)$ composed of four HM individuals and one HuB individual. The topology of Clade 3 which was also a distinct clade with a high posterior probability $(\mathrm{BPP}=100)$ composed of a resistant haplotype (E198A4) from $\mathrm{HeB}$ and two susceptible haplotypes from $\mathrm{HuB}$ was consistent with the research of Zhang et al. [21]. Corresponding with the BI tree, the topology of ML tree also showed the same result (not shown). To further test the multiple origins of the BZ resistance-associated SNP E198A (GCA) of the $H$. contortus worms from wild blue sheep, a network built by the neighbor-net method
(SplitsTrees) was constructed to discern their genetic relationships. The topology (Fig. 2) revealed that there was a clear boundary between the two haplotypes (E198A1 and E198A2) possessing BZ resistance-associated SNP E198A from wild blue sheep. Additionally, to detect the genetic diversity of $H$. contortus worms isolated from the wild blue sheep and other geographical regions in China, pairwise $F$ st value that is an index of genetic differentiation was calculated. A low pairwise Fst value (0.00774) was present between the worm groups from wild blue sheep and domestic sheep in FST. Similarly, low pairwise $F$ st values were also present between the wild blue sheep worm group and worm groups in other areas of China (Table 3).

\section{Discussion}

The present work is the first to monitor the three BZ resistance-associated SNPs, F167Y (TAC), E198A (GCA) and F200Y (TAC), in the isotype-1 $\beta$-tubulin gene in $H$. contortus worms from the wild blue sheep inhabiting the Helan Mountains. The BZ resistance-associated SNP E198A (GCA) was detected, whereas the other two SNPs, F167Y (TAC) and F200Y (TAC) were not found in the worms collected from wild blue sheep. In addition, for the $H$. contortus worms from the domestic sheep sympatric with the blue sheep, only heterozygosity at position 198 was detected (Table 1). It was shown that SNP E198A was dominant among the three BZ resistance-associated resistant SNPs in the two examined worm groups, which agreed with the argument proposed by other studies that SNP E198A (GCA) was most frequently encountered in China [21, 24]. The allele frequency of resistant at position 198 for the $H$. contortus

Table 2 Genetic diversity indices of isotype-1 $\beta$-tubulin gene of $H$. contortus worms collected from sympatric blue sheep and sheep in Helan Mountains, China

\begin{tabular}{lllllll}
\hline Host & N & No. of sequences & h & No. of haplotypes with E198A & Hd & $\pi$ \\
\hline Sheep & 20 & 20 & 10 & 0 & 0.926 & 0.03925 \\
Blue sheep & 27 & 27 & 16 & 2 & 0.952 & 0.04172 \\
Total & 47 & 47 & 24 & 2 & 0.952 & 0.04063 \\
\hline
\end{tabular}

Abbreviations: $\mathrm{N}, \mathrm{H}$. contortus population size; $h$, number of haplotypes; $\mathrm{Hd}$, haplotype diversity; $\pi$, nucleotide diversity 


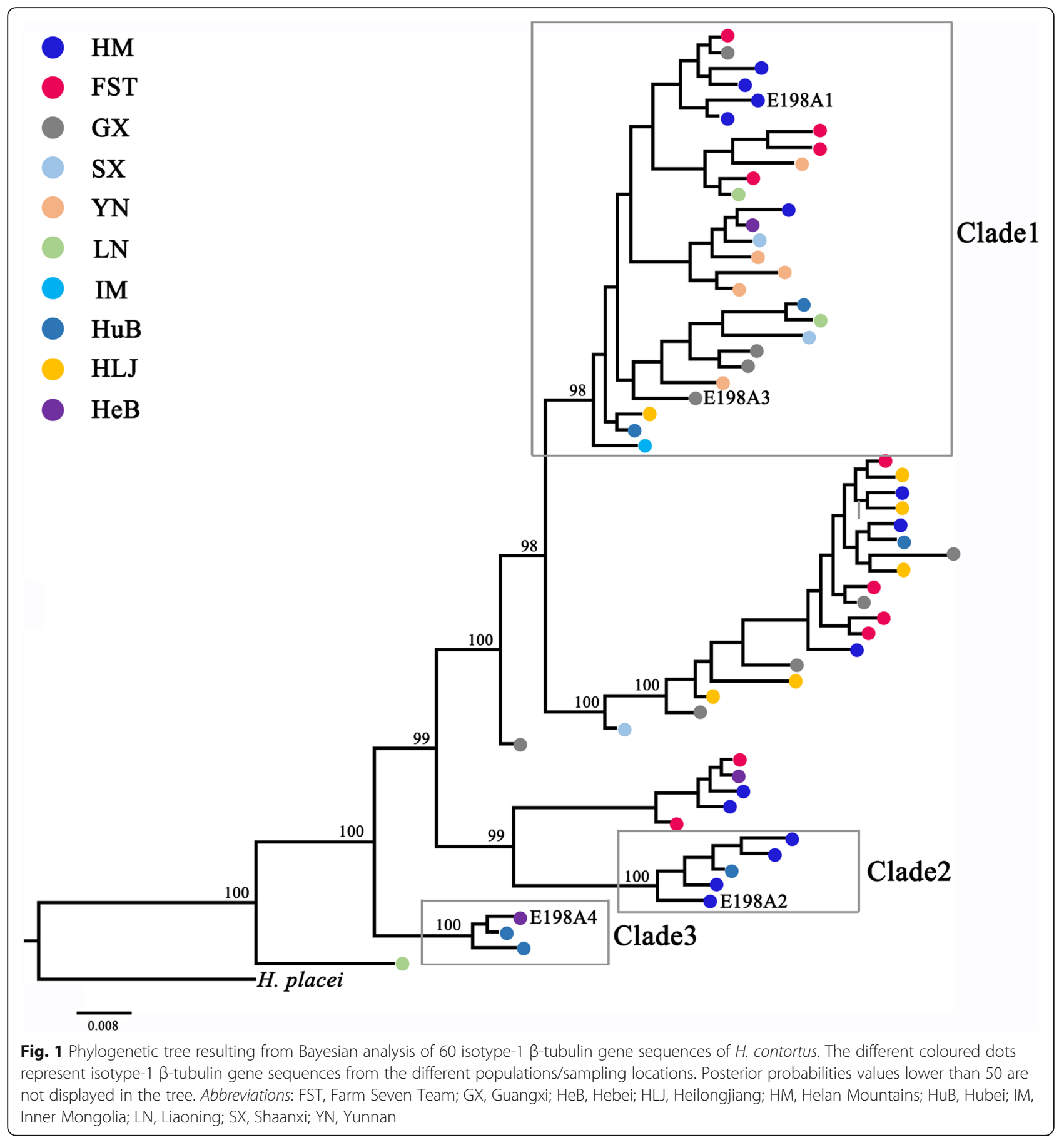

worms from blue sheep was $11.11 \%$ (Table 1), which was below the average frequency $(27.75 \%)$ of resistant at position 198 for the $H$. contortus worms collected from sheep and goats in eight geographical regions of China (GX, HLJ, IM, LN, SX, SZ, YD, YN) [21].

The results of our study revealed a high degree of genetic diversity within the two examined worm groups, which agreed with a previous study based on the mitochondrial gene [22]. Haplotype diversity of the isotype- $1 \beta$-tubulin sequences of $H$. contortus from the wild blue sheep and domestic sheep was at a high level $(0.952$ and 0.926 , respectively), which was similar to a previous study (from 0.455 to 0.939) in China [21]; similarly, the nucleotide diversity of the isotype- $1 \beta$-tubulin sequences of the two $H$. contortus groups was 0.03925 and 0.04172 , respectively (Table 2); these values are consistent with earlier studies on $H$. contortus from other geographical regions, such as Brazil (0.025-0.038) [11] and China (0.018-0.039) [21]. 


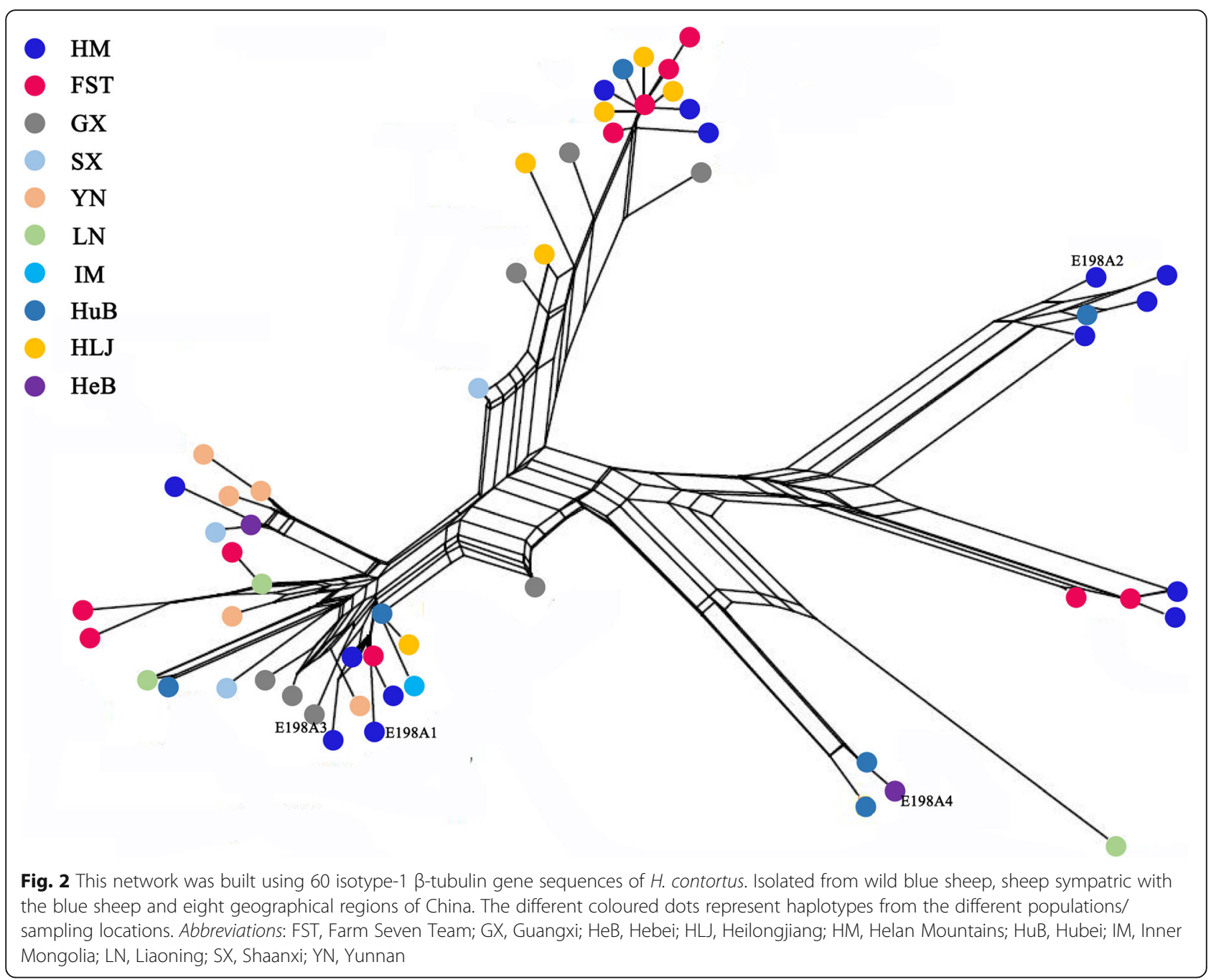

According to the topologies of the phylogenetic tree (Fig. 1) and the network (Fig. 2), the two haplotypes (E198A1 and E198A2) possessing resistant SNP E198A (GCA) from the wild blue sheep were distributed in two different groups and coupled to at least three susceptible haplotypes from the same worm group; this provided strong evidence that there were at least two independent origins of BZ resistance-associated E198A (GCA) within the $H$. contortus worms collected from the wild blue sheep. Resistant haplotype sequences E198A1 and E198A3 were contained in Clade 1 (Fig. 1), a distinct clade with high posterior probability $(\mathrm{BPP}=98)$. In contrast, the supported nodes of the subclades within Clade 1 were at a low level. The sequence similarity between E198A1 and E198A3 was 98.7\%. Hence, we hypothesized that one of the haplotypes (E198A1) possessing resistance-associated SNP E198A (GCA) from the wild blue sheep might have the same origin as the resistant haplotype (E198A3) from GX. The network built by the neighbor-net method also validated this hypothesis. Regarding another haplotype (E198A2) possessing resistance-associated SNP E198A (GCA) from wild blue sheep, the haplotype was shown to be clustered within three individuals from the wild blue sheep and one $\mathrm{HuB}$

Table 3 Pairwise Fst values of isotype-1 $\beta$-tubulin gene between $H$. contortus worm groups from the ten regions in China

\begin{tabular}{|c|c|c|c|c|c|c|c|c|c|}
\hline & FST & GX & $\mathrm{HeB}$ & $\mathrm{HLJ}$ & $\mathrm{HuB}$ & LN & IM & $S X$ & YN \\
\hline FST & - & 0.00714 & 0.00137 & 0.00767 & 0.01345 & 0.06312 & 0 & 0 & 0.19064 \\
\hline HM & 0.00774 & 0.09237 & 0 & 0.11089 & 0.00616 & 0.09620 & 0 & 0.03547 & 0.19749 \\
\hline
\end{tabular}

Bold indicates significant $F$ st values $(P<0.05)$

Abbreviations: FST Farm Seven Team, GX Guangxi, HeB Hebei, HL Heilongjiang, HM Helan Mountains, HuB Hubei, IM Inner Mongolia, LN Liaoning, SX Shaanxi; YN, Yunnan 
individual and formed a distinct clade (Clade 2) with high posterior probability $(\mathrm{BPP}=100)$. The network (Fig. 2) also revealed the same topology. Therefore, we hypothesized that this haplotype (E198A2) possessing resistance-associated SNP E198A (GCA) from the wild blue sheep had a different origin than the other three BZ resistance-associated E198A (GCA) SNPs in China. For E198A3 and E198A4, their distribution in the topologies of the tree and network was consistent with the study by Zhang et al. [11].

The evolutionary tree (Fig. 1) using the isotype-1 $\beta$-tubulin gene of $H$. contortus showed the same result as a previous phylogenetic analysis based on mitochondrial DNA [22]: there was no obvious boundary among these $H$. contortus worms according to geographical origin and that the haplotype sequences of $H$. contortus from wild blue sheep were randomly dispersed within the main clades of the tree. Furthermore, this phenomenon was consistent with the topology of the network (Fig. 2). Additionally, low Fst values (Table 3) were present among all the $H$. contortus worms collected from different regions in China. All these results indicated that a high level of gene flow without obvious geographical barriers was present among all the $H$. contortus worms in China, including the $H$. contortus worms from wild blue sheep.

The BZ resistance-associated SNP E198A (GCA) was found in the blue sheep $H$. contortus worms, which was a question worthy of consideration why the $H$. contortus worms collected from wild hosts revealed presence of markers for anthelmintic resistance. The Helan Mountains is more than just a natural habitat of wild blue sheep, it was also a main pastoral area of Ningxia Province in China. Every year, approximately 100,000 sheep were observed on the mountains from June to August [25], and the BZ resistance in China has a high prevalence. In current work, a high level of gene flow was detected based on the isotype-1 $\beta$-tubulin gene among the $H$. contortus worms collected from wild and domestic hosts, which was consistent with a previous study based on mitochondrial DNA [22]. The gene flow is determined by the life histories of parasite, host movement, effective population sizes and multiple host species being reared together in common grazing pastures [26]. Therefore, we assumed that the high level of gene flow among the $H$. contortus worms from wild and domestic hosts was caused by the sympatric grazing. Consequently, BZ resistance mutations of $H$. contortus from domestic hosts were spread into this wild hosts $H$. contortus worms because of the sympatric grazing. Results of current work revealed that one of the haplotypes (E198A1) possessing resistance-associated SNP E198A (GCA) from the wild blue sheep might have the same origin as the resistant haplotype (E198A3) from GX, which validated this hypothesis.

\section{Conclusions}

The present study is the first to monitor the three BZ resistance-associated SNPs F167Y (TAC), E198A (GCA) and F200Y (TAC) in the isotype-1 $\beta$-tubulin gene in $H$. contortus worms from the wild blue sheep inhabiting Helan Mountains. BZ resistance-associated SNP E198A (GCA) was detected and the frequency of homozygous resistant was $7.40 \%$, whereas another two SNPs F167Y (TAC) and F200Y (TAC) were not found. Genetic relationships analysis revealed that the two haplotypes (E198A1 and E198A2) possessing resistant SNP E198A from the wild blue sheep had two different independent origins.

\section{Methods}

\section{Parasite material and DNA extraction}

Wild blue sheep is widely distributed and has no any natural predators in the Helan Mountains. To control population sizes of the blue sheep, local government agents used the tranquilizer guns to cull a few of old and sick individuals that were eliminated naturally by blue sheep populations. In current work, approved by the local government agents, twenty-seven adult $H$. contortus were harvested from three wild blue sheep that were culled from the population by them in the areas of Qingyang Ravine and Xiazi Ravine. Another six domestic sheep that were slaughtered by herdsmen in their daily life were randomly chosen from a mountain village (Farm Seven Team) near the natural habitat of blue sheep. And we harvested twenty adult $H$. contortus from these domestic sheep with approval of the owners. All experimental designs and animals handling were approved by the Institutional Animal Care and Use Committee of Northeast Forestry University. Detailed information about the sampling sites of the hosts was shown in Fig. 3. Worm DNA was extracted using a QIAamp DNA kit (QIAamp, Hilden, Germany) following the manufacturer's directions and then stored at $-20^{\circ} \mathrm{C}$. All worm DNA was used to assess the BZ-associated SNPs. As described by Zhang et al. [21], two pooled DNA samples representing the two $H$. contortus populations from the wild and domestic hosts were constructed to analyze the genetic diversity.

\section{PCR amplification and sequencing to determine the} frequencies of the isotype-1 $\beta$-tubulin gene F167Y, E198A and F200Y BZ resistance-associated SNPs in $H$. contortus A region $(\sim 375 \mathrm{bp})$ that included exons 4 and 5 and their intervening intron of the isotype- $1 \beta$-tubulin gene was amplified using nested PCR with newly designed primers that flanked the three BZ-associated SNPs. The primers were designed using Oligo 7 software [27] according to the complete $\beta$-tubulin sequence of $H$. contortus (GenBank accession no. X67489). Primers BR_F1 (5'-AGGGAGCCGAGCTAGTTGATA-3') and BR_R1 


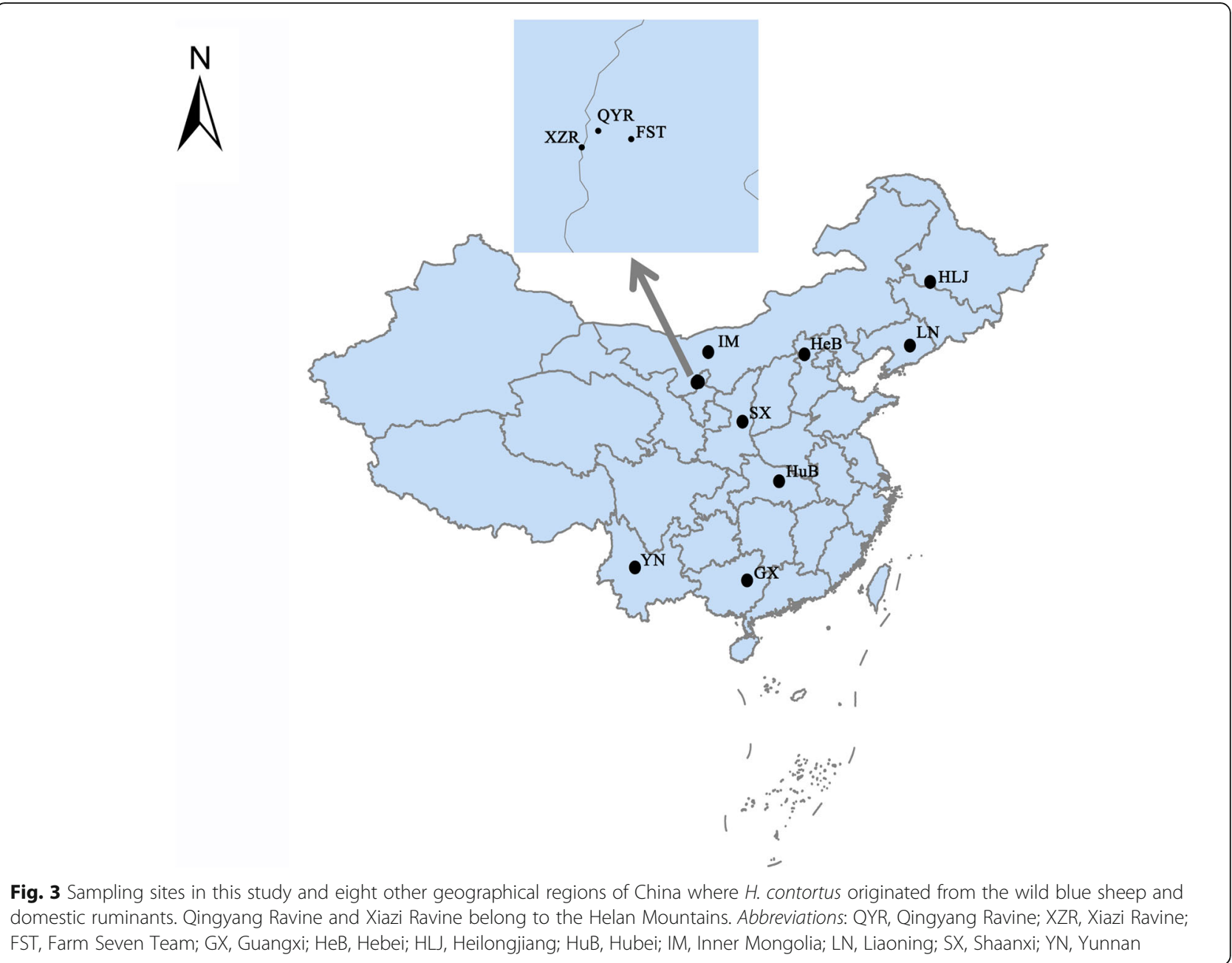

(5' -AAGTGAAGACGAGGGAATGGA-3') were used in the first-step PCR amplification, and the reactions $(25 \mu \mathrm{l})$ included $2.5 \mu \mathrm{l}$ of PCR buffer, $2.5 \mu \mathrm{l}$ of dNTPs, $2 \mu \mathrm{l}$ of $\mathrm{MgCl}_{2}, 3 \mu \mathrm{l}$ of DNA, $1 \mu \mathrm{l}$ of each primer, $12 \mu \mathrm{l}$ of $\mathrm{dd}_{2} \mathrm{O}$ water and $1 \mu \mathrm{l}$ of Thermo Scientific Taq DNA Polymerase under the following conditions: $2 \mathrm{~min}$ at $94^{\circ}$ C, 30 cycles of $40 \mathrm{~s}$ at $94{ }^{\circ} \mathrm{C}, 30 \mathrm{~s}$ at $54^{\circ} \mathrm{C}, 1 \mathrm{~min}$ at $72^{\circ} \mathrm{C}$; and $7 \mathrm{~min}$ at $72^{\circ} \mathrm{C}$. Forward primer BR_F2 (5'-CTTG GAGGAGGCACTGGAT-3') and reverse primer BR_R2 (5'-GTGAAGACGAGGGAATGGA-3') were used for the second-step (nested) PCR amplification, and the reactions $(25 \mu \mathrm{l})$ were performed in $2.5 \mu \mathrm{l}$ of PCR buffer, $2.5 \mu \mathrm{l}$ of dNTPs, $2 \mu \mathrm{l}$ of $\mathrm{MgCl}_{2}, 2 \mu \mathrm{l}$ of first-step PCR amplification, $1 \mu \mathrm{l}$ of each primer, $13 \mu \mathrm{l}$ of $\mathrm{dd}_{2} \mathrm{O}$ water and $1 \mu \mathrm{l}$ of Thermo Scientific Taq DNA Polymerase under the following conditions: $94^{\circ} \mathrm{C}$ for $2 \mathrm{~min}, 30$ cycles of $30 \mathrm{~s}$ at $94{ }^{\circ} \mathrm{C}, 15 \mathrm{~s}$ at $55^{\circ} \mathrm{C}, 30 \mathrm{~s}$ at $72^{\circ} \mathrm{C}$; and $72{ }^{\circ} \mathrm{C}$ for $7 \mathrm{~min}$. Five microliters of nested PCR product was examined on a $1.0 \%$ agarose gel to verify that the product contained a single band of the appropriate size. Column-purified nested PCR products were sent to BGI
(Beijing, China) for sequencing in forward and reverse directions with the primers BR_F2 and BR_R2.

To assess the relative frequencies of the BZ resistanceassociated SNPs, ChromasPro version 1.5 software was used to trace the amplifications and mainly focused on positions 167, 198 and 200 according to the threshold described by Kotze et al. [19]. Relative allelic frequencies and genotypic frequencies were calculated according to Tiwari et al. [28] and the 95\% confidence intervals of these frequencies were estimated as Wilson-Score intervals using the binom-wilson tool from the epitools package in the statistical programming language $R$.

Amplification, cloning and sequencingof isotype-1 $\beta$ tubulin gene to detect the BZ resistance-associated SNPs F167Y, E198A and F200Y

A partial fragment of the isotype-1 $\beta$-tubulin gene was amplified from the two pooled DNA samples, and the nested PCR products were purified and then cloned into Escherichia coli DH5 $\alpha$ using the pMD19-T Easy Vector System (TaKaRa) following the manufacturer's protocols. 
Twenty and 27 clones were selected from the $H$. contortus worms from domestic sheep and wild blue sheep, respectively, and sequenced in both directions (forward and reverse). The published isotype- $1 \quad \beta$-tubulin gene (GenBank accession no. X67489) was used as the reference sequence to detect the SNPs F167Y, E198A and F200Y.

\section{Sequence diversity and phylogenetic analyses}

All the raw sequences (isotype- $1 \beta$-tubulin gene) were aligned using ClustalX v2.0 software [29]. The haplotypes were produced using the DnaSP 5.10 software [30]; in addition, haplotype diversity and nucleotide diversity were also calculated through this software. To define the spread and origins of BZ resistance-associated SNPs in $H$. contortus from wild blue sheep, another 36 isotype-1 $\beta$-tubulin gene haplotype sequences of $H$. contortus (Additional file 1) isolated from sheep and goats in eight geographical regions of China (Fig. 1) were retrieved from the NCBI nucleotide database (https:// www.ncbi.nlm.nih.gov/) to construct phylogenetic trees. Phylogenetic analyses were hypothesized using maximum likelihood (ML) and Bayesian inference (BI). The partial $\beta$-tubulin gene of Haemonchus placei (GenBank accession no. KJ598498) acted as the outgroup. For all the isotype- $1 \beta$-tubulin gene sequences, multiple alignments were produced using ClustalX v2.0 software [29], and ambiguously aligned regions were excluded using Gblocks-0.91 [31]. The program Modeltest 3.7 [32] was used to select the appropriate model of nucleotide substitution according to Akaike's information criterion (AIC). ML analyses were conducted using raxmlGUI v1.5 [33], and the best-fitting nucleotide substitution model was GTR + I + G. Bootstrap branch support values (MLBS) were obtained with 1000 rapid bootstrap inferences and subsequently sought in a thorough ML search of the data set. BI analyses were hypothesized using MrBayes v3.2 [34], and the best-fitting models for the partial isotype- $1 \beta$-tubulin sequence evolution was GTR $+\mathrm{I}+\mathrm{G}$. The following parameters were applied: four Markov chain Monte Carlo (MCMC) were run for 2 runs from random starting trees for 10 million generations, and trees were sampled every 100 generations; 25,000 generations were discarded as "burn-in", and the remaining samples were used to calculate Bayesian posterior probabilities (BPP). Topologies of phylogenetic trees were drawn using FigTree v1.4.2 software (http://tree.bio.ed.ac.uk/software/figtree). For population genetic analysis, the program Arlequin 3.5 [35] was used to calculate the pairwise Fst value. In addition, network based on genetic distance were generated using the neighbor-net method in the program SplitsTrees v 4.0 [36].

\section{Additional files}

Additional file 1: Table S1. Information on 24 different isotype-1 $\beta$ tubulin gene haplotypes from two $H$. contortus groups isolated form sympatric blue sheep and sheep. (DOC $13 \mathrm{~kb}$ )

Additional file 2: Table S2. Information on 36 different isotype-1 $\beta$-tubulin gene haplotypes from eight $H$. contortus groups in China. (DOC 14 kb)

\section{Abbreviations \\ AIC: Akaike's information criterion; BI: Bayesian inference; BPP: Bayesian posterior probability; BZs: Benzimidazoles; Cl: Confidence interval; FST: Farm Seven Team; GX: Guangxi; HLJ: Heilongjiang; HMB: Wild blue sheep inhabiting in Helan Mountains; IM: Inner Mongolia; LN: Liaoning; MJ: Median- joining; ML: Maximum likelihood; QYR: Qingyang Ravine; SX: Shaanxi; SZ: Suizhou; TBR: Tree bisection reconnection; XZR: Xiazi Ravine; YD: Yidu; YN: Yunnan}

\section{Acknowledgments}

Not applicable.

\section{Funding}

This work was supported by the following Grants: National Key Research and Development Program (project 2017YFD0501702), Fundamental Research Funds for the Central Universities (2572018BE07) and Surveillance of Wildlife Diseases from the State Forestry Administration of China. The funding body was solely involved in funding and had no role in the design of the study, the collection, analysis, and interpretation of the data, or in writing the manuscript.

\section{Availability of data and materials}

The datasets supporting the findings of this article are included within the article. GenBank accession numbers of 24 isotype- $1 \beta$-tubulin gene haplotype sequences of $\mathrm{H}$. contortus isolated from wild blue sheep are MH359364 MH359387 (Additional file 1); GenBank accession numbers of the eight geographical H. contortus populations are shown in Additional file 2.

\section{Authors' contributions}

DS carried out laboratory work and wrote the manuscript. ZP and MH performed the data analyses. ZP and ZZ isolated $\mathrm{H}$. contortus worms from the wild and domestic sheep. $\mathrm{ZH}$ helped in conceived of this work and participated in its design. ZL contributed in manuscript revision. All authors read and approved the final manuscript.

\section{Ethics approval and consent to participate}

We declare that all the wild blue sheep in current work were culled from the population by local Helan Mountains government agents. There is no conflict of interest with species conservation guidelines. Collecting the $\mathrm{H}$. contortus worms from the wild sheep was approved by the local Helan government agents. All experimental designs and animals handling were approved by the Institutional Animal Care and Use Committee of Northeast Forestry University.

\section{Consent for publication}

Not applicable.

\section{Competing interests}

The authors declare that they have no competing interests.

\section{Publisher's Note}

Springer Nature remains neutral with regard to jurisdictional claims in published maps and institutional affiliations.

\section{Author details}

${ }^{1}$ College of Wildlife Resources, Northeast Forestry University, Harbin, China. ${ }^{2}$ Key Laboratory of Wildlife Conservation, China State Forestry Administration, Harbin, China. ${ }^{3}$ State Key Laboratory of Agricultural Microbiology, College of Veterinary Medicine, Huazhong Agricultural University, Wuhan, China. 
Received: 30 October 2018 Accepted: 6 March 2019

Published online: 12 March 2019

\section{References}

1. Anderson TJ, Blouin MS, Beech RN. Population biology of parasitic nematodes: applications of genetic markers. Adv Parasitol. 1998;41(1):219-27.

2. Prichard R. Genetic variability following selection of Haemonchus contortus with anthelmintics. Trends Parasitol. 2001;17(9):445-53.

3. Nikolaou S, Gasser RB. Prospects for exploring molecular developmental processes in Haemonchus contortus. Int J Parasitol. 2006;36(8):859-68.

4. Gasser RB, Bott NJ, Chilton NB, Hunt P, Beveridge I. Toward practical, DNAbased diagnostic methods for parasitic nematodes of livestock - bionomic and biotechnological implications. Biotechnol Adv. 2008;26(4):325-34.

5. Garretson PD, Hammond EE, Craig TM, Holman PJ. Anthelmintic resistant Haemonchus contortus in a giraffe (Giraffa camelopardalis) in Florida. J Zoo Wildl Med. 2009;40(1):131-9.

6. Coop RL, Martin RJ, Schallig HDFH, Chappell LH. The development of anthelmintic resistance in sheep nematodes. Parasitology. 2000;120(7):S95-107.

7. Silvestre A, Cabaret J. Mutation in position 167 of isotype 1 beta-tubulin gene of Trichostrongylid nematodes: role in benzimidazole resistance? Mol Biochem Parasitol. 2002;120(2):297-300.

8. Ghisi M, Kaminsky R, Mäser P. Phenotyping and genotyping of Haemonchus contortus isolates reveals a new putative candidate mutation for benzimidazole resistance in nematodes. Vet Parasitol. 2007;144(3):313-20.

9. Kwa MSG, Veenstra JG, Dijk MV, Roos MH. $\beta$-Tubulin genes from the parasitic nematode Haemonchus contortus modulate drug resistance in Caenorhabditis elegans. J Mol Biol. 1995;246(4):500-10.

10. Kotze AC, Hunt PW, Skuce P, Samson-Himmelstjerna GV, Martin RJ, Sager H, Krücken J, Hodgkinson J, Lespine A, Jex AR. Recent advances in candidategene and whole-genome approaches to the discovery of anthelmintic resistance markers and the description of drug/receptor interactions. Int J Parasitol Drugs Drug Resist. 2014;4(3):164-84.

11. Brasil BSAF, Nunes RL, Bastianetto E, Drummond MG, Carvalho DC, Leite RC, Molento MB, Oliveira DAA. Genetic diversity patterns of Haemonchus placei and Haemonchus contortus populations isolated from domestic ruminants in Brazil. Int J Parasitol. 2012;42(5):469-79.

12. Barrère V, Keller K, Samson-Himmelstjerna GV, Prichard RK. Efficiency of a genetic test to detect benzimidazole resistant Haemonchus contortus nematodes in sheep farms in Quebec. Canada Parasitol Int. 2013;62(5): 464-70.

13. Barrere V, Falzon LC, Shakya KP, Menzies PI, Peregrine AS, Prichard RK. Assessment of benzimidazole resistance in Haemonchus contortus in sheep flocks in Ontario, Canada: comparison of detection methods for drug resistance. Vet Parasitol. 2013;198(1-2):159-65.

14. Barrère V, Alvarez L, Suarez G, Ceballos L, Moreno L, Lanusse C, Prichard RK. Relationship between increased albendazole systemic exposure and changes in single nucleotide polymorphisms on the $\beta$-tubulin isotype 1 encoding gene in Haemonchus contortus. Vet Parasitol. 2012;186(3-4):344-9.

15. Chaudhry U, Miller M, Yazwinski T, Kaplan R, Gilleard J. The presence of benzimidazole resistance mutations in Haemonchus placei from US cattle. Vet Parasitol. 2014;204(3-4):411-5

16. Santos JMLD, Monteiro JP, Ribeiro WLC, Macedo ITF, Vieira LDS, Bevilaqua CML. Identification and quantification of benzimidazole resistance polymorphisms in Haemonchus contortus isolated in northeastern Brazil. Vet Parasitol. 2014;199(3-4):160-4.

17. Elizabeth R, Fiona W, Andrew T, Charlotte B, Yvonne B, Philip John S, Frank J, John SG. The emergence of resistance to the benzimidazole anthlemintics in parasitic nematodes of livestock is characterised by multiple independent hard and soft selective sweeps. PLoS Negl Trop Dis. 2015;9(2):e0003494.

18. Rufener L, Kaminsky R, Mäser P. In vitro selection of Haemonchus contortus for benzimidazole resistance reveals a mutation at amino acid 198 of $\beta$ tubulin. Mol Biochem Parasitol. 2009;168(1):120-2.

19. Kotze AC, Cowling K, Bagnall NH, Hines BM, Ruffell AP, Hunt PW, Coleman GT. Relative level of thiabendazole resistance associated with the E198A and F200Y SNPs in larvae of a multi-drug resistant isolate of Haemonchus contortus. Int J Parasitol Drugs Drug Resist. 2012;2(12):92-7.

20. Skuce P, Stenhouse L, Jackson F, Hypša V, Gilleard J. Benzimidazole resistance allele haplotype diversity in United Kingdom isolates of Teladorsagia circumcincta supports a hypothesis of multiple origins of resistance by recurrent mutation. Int J Parasitol. 2010;40(11):1247-55.
21. Zhang Z, Gasser RB, Yang X, Yin F, Zhao G, Bao M, Pan B, Huang W, Wang C, Zou F. Two benzimidazole resistance-associated SNPs in the isotype-1 $\beta$ tubulin gene predominate in Haemonchus contortus populations from eight regions in China. Int J Parasitol Drugs Drug Resist. 2016;6(3):199-206.

22. Shen DD, Wang JF, Zhang DY, Peng ZW, Yang TY, Wang ZD, Bowman DD, Hou ZJ, Liu ZS. Genetic diversity of Haemonchus contortus isolated from sympatric wild blue sheep (Pseudois nayaur) and sheep in Helan Mountains. China Parasit Vectors. 2017;10(1):437.

23. Cai KZ, Yang XY, Wang XL, Hao C, Gui-Hua MA, Yang FB, Fang YB, Zong XB, Ren DX, Liu ZL. Investigation on resistance of gastrointestinal nematodes in sheep and goats to anthelmintics in Ningxia. China China Vet Sci. 2007;2: 1120-3 (In Chinese).

24. Bo X. Multiplex PCR detection of allel on benzimidazole-resistance or susceptibity in natural populations of Haemonchus contortus. Zhongguo Nongye Kexue. 2005;32(1):47-51 (In Chinese).

25. Wang XM, Liu ZX, Li M. L YG: the blue sheep population ecology and its conservation in Helan Mountain. China Chinese Biodivers. 1998:6:1-5 (In Chinese).

26. Hussain T, Periasamy K, Nadeem A, Babar ME, Pichler R, Diallo A. Sympatric species distribution, genetic diversity and population structure of Haemonchus isolates from domestic ruminants in Pakistan. Vet Parasitol. 2014:206(3-4):188-99.

27. Rychlik W. OLIGO 7 primer analysis software. Methods Mol Biol. 2007;402: 35-60.

28. Tiwari J, Kumar S, Kolte AP, Swarnkar CP, Singh D, Pathak KM. Detection of benzimidazole resistance in Haemonchus contortus using RFLP-PCR technique. Vet Parasitol. 2006;138(3):301-7.

29. Thompson JD, Gibson TJ, Plewniak F, Jeanmougin F, Higgins DG. The CLUSTAL_X windows interface: flexible strategies for multiple sequence alignment aided by quality analysis tools. Nucleic Acids Res. 1997;25(25): 4876-82.

30. Librado P, Rozas J. DnaSP v5: a software for comprehensive analysis of DNA polymorphism data. Bioinformatics. 2009:25(11):1451-2.

31. Talavera G, Castresana J. Improvement of phylogenies after removing divergent and ambiguously aligned blocks from protein sequence alignments. Syst Biol. 2007;56(4):564-77.

32. Posada D, Crandall KA. MODELTEST: testing the model of DNA substitution. Bioinformatics. 1998;14(9):817-8.

33. Silvestro D, Michalak I. raxm|GUI: a graphical front-end for RAxML. Org Divers Evol. 2012;12(4):335-7.

34. Ronquist F, Huelsenbeck JP. MrBayes 3: Bayesian phylogenetic inference under mixed models. Bioinformatics. 2003:19(12):1572-4

35. Excoffier L, Lischer HE. Arlequin suite ver 3.5: a new series of programs to perform population genetics analyses under Linux and windows. Mol Ecol Resour. 2010;10(3):564-7.

36. Huson DH, Bryant D. Application of phylogenetic networks in evolutionary studies. Mol Biol Evol. 2006;23:254-67.

Ready to submit your research? Choose BMC and benefit from:

- fast, convenient online submission

- thorough peer review by experienced researchers in your field

- rapid publication on acceptance

- support for research data, including large and complex data types

- gold Open Access which fosters wider collaboration and increased citations

- maximum visibility for your research: over $100 \mathrm{M}$ website views per year

At BMC, research is always in progress.

Learn more biomedcentral.com/submissions 